M. Nakai and T. Tada

Nagoya Math. J.

Vol. 86 (1982), 85-99

\title{
DIRICHLET INTEGRAL AND PICARD PRINCIPLE
}

\author{
MITSURU NAKAI AND TOSHIMASA TADA
}

A density $P$ on the punctured unit disk $\Omega: 0<|z|<1$ is a 2 -form $P(z) d x d y$ whose coefficient $P(z)$ is a real valued nonnegative locally Hölder continuous function on the closed punctured unit disk $\bar{\Omega}: 0<|z| \leqq 1$. Here we consider $\Omega$ as an end of the punctured sphere $0<|z| \leqq+\infty$ so that the point $z=0$ is viewed as the ideal boundary $\delta \Omega$ of $\Omega$ and the unit circle $|z|=1$ as the relative boundary $\partial \Omega$ of $\Omega$. We denote by $\mathscr{D}=$ $\mathscr{D}(\Omega)$ the family of densities on $\Omega$. A density $P$ on $\Omega$ gives rise to an elliptic operator $L=L_{P}$ on $\bar{\Omega}$ defined by

$$
L u=L_{P} u=\Delta u-P u, \quad \Delta=\partial^{2} / \partial x^{2}+\partial^{2} / \partial y^{2} .
$$

Since $\delta \Omega$ is of parabolic character, there exists a unique bounded solution $e=e_{P}$, referred to as the $P$-unit on $\Omega$, of $L u=0$ on $\Omega$ with continuous boundary values 1 on $\partial \Omega$. With the operator $L=L_{P}$ we associate an elliptic operator $\hat{L}=\hat{L}_{P}$, referred to as the associate operator to $L$, given by

$$
\hat{L} v=\hat{L}_{P} v=\Delta v+2 \nabla \log e_{P} \cdot \nabla v, \quad \nabla=(\partial / \partial x, \partial / \partial y) .
$$

We denote by $\mathscr{P}=\mathscr{P}_{P}$ the family of nonnegative solutions $u$ of $L u=0$ on $\Omega$ with vanishing boundary values on $\partial \Omega$, by $\mathscr{B}=\mathscr{B}_{P}$ the family of bounded solutions $u$ of $L u=0$ on $\Omega$ and similarly, by $\hat{\mathscr{B}}_{=}=\hat{\mathscr{B}}_{P}$ the family of bounded solutions $v$ of $\hat{L} v=0$ on $\Omega$.

We are particularly interested in those densities $P$ for which $\mathscr{P}=\mathscr{P}_{P}$ is generated by a single element $u_{0}: \mathscr{P}=\left\{\lambda u_{0} ; \lambda \in \boldsymbol{R}^{+}\right\}$, where $\boldsymbol{R}$ is the real number field and $\boldsymbol{R}^{+}$is the set of nonnegative real numbers. Since $P \equiv$ 0 is the typical one of this character found by Picard, we say, after Bouligand (cf. Brelot [2]), that the Picard principle is valid for $P$ at $\delta \Omega$ if $\mathscr{P}_{P}$ is generated by a single element, and we denote by $\mathscr{D}_{\mathfrak{\beta}}=\mathscr{D}_{\mathfrak{R}}(\Omega)$ the family of densities on $\Omega$ for which the Picard principle is valid. It is a fasci-

Received October 20, 1979.

Both of the authors were supported by Grant-in-Aid for Scientific Research, The Japanese Ministry of Education, Science and Culture. 
nating problem to characterize the family $\mathscr{D}_{\mathfrak{p}}$. We compile some of papers answering to this question partially at the end of this paper. If the limit $\lim _{z \rightarrow \delta \Omega} u(z)$ exists for every $u$ in $\hat{\mathscr{B}}_{P}$, then we say that the (weak) Riemann theorem is valid for the operator $\hat{L}_{P}$. We denote by $\mathscr{D}_{\mathfrak{R}}$ the family of densities $P$ such that the Riemann theorem is valid for $\hat{L}_{P}$. We have the following duality theorem (cf. Heins [9], Hayashi [8], [26]):

$$
\mathscr{D}_{\Re}=\mathscr{D}_{\mathfrak{R}} \text {. }
$$

Therefore characterizing $\mathscr{D}_{\mathfrak{F}}$ is identical with characterizing $\mathscr{D}_{\mathfrak{N}}$. There are quite a few instances that the Dirichlet integral plays very important role to single out densities in $\mathscr{D}_{\mathfrak{R}}$ among $\mathscr{D}$.

The purpose of this paper is to clarify the efficiency of Dirichlet integrals and at the same time its limitation in the study of the Picard principle. For the purpose we further classify $\mathscr{D}$. A density $P$ is said to be finite if

$$
\int_{\Omega} P(z) d x d y<+\infty
$$

and we denote by $\mathscr{D}_{1}$ the family of finite densities on $\Omega$. The importance of the class $\mathscr{D}_{1}$ lies in the fact that $\mathscr{D}_{1} \subset \mathscr{D}_{\mathfrak{B}}$ (cf. [27], Kawamura [13]). In connection with the class $\mathscr{D}_{1}$, we consider the class $\mathscr{D}_{\mathscr{C D}}$ of, what we call, densities $P$ of strongly $D$-type characterized by

$$
\int_{0}\left|\nabla \log e_{P}(z)\right|^{2} d x d y<+\infty .
$$

It is known that $\mathscr{D}_{1} \subset \mathscr{D}_{\subseteq D}$ (cf. [27]). It is easy to see that the Dirichlet integral $D_{\{0<|z|<r\}}(u)$ of any $u$ in $\mathscr{B}_{P}$ is finite:

$$
D_{\{0<|z|<r\}}(u)=\int_{0<|z|<r}|\nabla u(z)|^{2} d x d y<+\infty
$$

for every $r$ in $(0,1)$. The same may or may not be true for the class $\hat{\mathscr{B}}_{P}$. If

$$
D_{\{0<|z|<r\}}(v)=\int_{0<|z|<r}|\nabla v(z)|^{2} d x d y<+\infty
$$

for any $v$ in $\hat{\mathscr{B}}_{P}$ and any $r$ in $(0,1)$, then we say that $P$ is of $D$-type, and we denote by $\mathscr{D}_{D}$ the family of densities of $D$-type on $\Omega$. We know (cf. [27]) that $\mathscr{D}_{\subseteq D} \subset \mathscr{D}_{\mathbb{D}} \subset \mathscr{D}_{\mathfrak{R}}$ from which we deduced the relation $\mathscr{D}_{1} \subset \mathscr{D}_{\mathfrak{p}}$. Therefore it has been known that $\mathscr{D}_{1} \subset \mathscr{D}_{\text {ED }} \subset \mathscr{D}_{\mathbb{D}} \subset \mathscr{D}_{\mathfrak{M}}=\mathscr{D}_{\mathfrak{P}}$. We will 
study whether these inclusions are proper or not. The conclusion will be the following:

$$
\mathscr{D}_{1}=\mathscr{D}_{\overparen{D D}}<\mathscr{D}_{\mathscr{D}}<\mathscr{D}_{\mathfrak{R}}=\mathscr{D}_{\mathfrak{R}},
$$

where $<$ indicates the strict inclusion.

In $\S 1$ we will prove $\mathscr{D}_{1}=\mathscr{D}_{\text {ธD }}$ by establishing an identity evaluating the Dirichlet integral of $\log e_{P}$ in terms of the integral involving $P$. In $\$ 2$ a necessary and sufficient condition is given for a rotation free density $P$ to belong to $\mathscr{D}_{\mathfrak{D}}$. Here a density $P$ is rotation free, by definition, if $P(z)$ $=P(|z|)$ for every $z$ in $\Omega$. As an application of the result in $\S 2$, we will see in $\S 3$ that the simple density $P(z)=|z|^{-2}$ belongs to $\mathscr{D}_{\mathscr{D}}-\mathscr{D}_{\odot \triangleright}$ and $P(z)=|z|^{-2}(\log |z|)^{2}$ belongs to $\mathscr{D}_{\Re}-\mathscr{D}_{\mathscr{D}}$. Actually, as we will see in $\S 3$, belonging to $\mathscr{D}_{\mathscr{D}}$ is very delicate:

$$
\begin{cases}c|z|^{-2}(\log |z|)^{2} \in \mathscr{D}_{\triangleright} & \text { for } c \in[0,1), \\ c|z|^{-2}(\log |z|)^{2} \notin \mathscr{D}_{\triangleright} & \text { for } c \in[1,+\infty) .\end{cases}
$$

\section{§1. An identity}

1. Consider a subregion $S$ of $\Omega$ with its relative boundary $\partial S$ of a simple closed curve in $\Omega$ and with the ideal boundary $z=0$. We do not exclude the case $S=\Omega$ so that $\partial S=\partial \Omega$. For every closed punctured disk $\bar{V}_{\varepsilon}: 0<|z| \leqq \varepsilon$ contained in $S$, we denote by $w_{s}$ the harmonic measure of $\partial S$ considered on $S-\bar{V}_{\varepsilon}$. Then the Stokes formula yields

$$
\begin{aligned}
\int_{\partial S} \frac{\partial e(z)}{\partial n} d s & =\int_{\partial\left(S-\bar{V}_{s}\right)} w_{s}(z) \frac{\partial e(z)}{\partial n} d s \\
& =\int_{S-\bar{V}_{s}} \nabla w_{s}(z) \cdot \nabla e(z) d x d y+\int_{S-\bar{V}_{s}} w_{s}(z) \Delta e(z) d x d y,
\end{aligned}
$$

where $\partial / \partial n$ is the outer normal derivative and $d s$ the line element. By the maximum principle and the Harnack principle, we see that $w_{\mathrm{s}} \uparrow 1$ uniformly on each compact subset of $S \cup \partial S$. On setting $w_{s}=0$ on $\bar{V}_{s}$, a simple application of the Stokes formula yields

$$
\int_{S}\left|\nabla\left(w_{\varepsilon}-w_{\varepsilon^{\prime}}\right)(z)\right|^{2} d x d y=\int_{S}\left|\nabla w_{\iota}(z)\right|^{2} d x d y-\int_{S}\left|\nabla w_{\varsigma^{\prime}}(z)\right|^{2} d x d y
$$

for $\varepsilon<\varepsilon^{\prime}$. Hence in particular we see that

$$
\int_{S}\left|\nabla w_{\iota}(z)\right|^{2} d x d y \downarrow 0 \quad(\varepsilon \downarrow 0)
$$


By the Schwarz inequality

$$
\left(\int_{S-\bar{V}_{\varepsilon}} \nabla w_{\varepsilon}(z) \cdot \nabla e(z) d x d y\right)^{2} \leqq \int_{S-\bar{V}_{\varepsilon}}\left|\nabla w_{\varepsilon}(z)\right|^{2} d x d y \cdot \int_{S-\bar{V}_{\varepsilon}}|\nabla e(z)|^{2} d x d y
$$

Since the Dirichlet integral of any function in $\mathscr{B}_{P}$ is finite,

$$
\int_{S-\bar{V}_{\varepsilon}}|\nabla e(z)|^{2} d x d y
$$

is dominated by

$$
\int_{\Omega}|\nabla e(z)|^{2} d x d y<+\infty
$$

Thus we may conclude that

$$
\lim _{\varepsilon \rightarrow 0} \int_{S-\bar{V}_{\varepsilon}} \nabla w_{\varepsilon}(z) \cdot \nabla e(z) d x d y=0
$$

Observe that

$$
\int_{S-\bar{V}_{\varepsilon}} w_{\epsilon}(z) \Delta e(z) d x d y=\int_{S} w_{\epsilon}(z) e(z) P(z) d x d y .
$$

The Lebesgue-Fatou theorem implies that

$$
\lim _{\varepsilon \rightarrow 0} \int_{S-\bar{\nabla}_{\varepsilon}} w_{\varepsilon}(z) \Delta e(z) d x d y=\int_{S} e(z) P(z) d x d y .
$$

We finally conclude that

$$
\int_{\partial S} \frac{\partial e(z)}{\partial n} d s=\int_{S} e(z) P(z) d x d y
$$

This means that $e(z) P(z) d x d y$ is a finite measure on $\Omega$.

2. Consider a continuous function $f$ on $\partial S$. We denote by $H_{f}^{S}$ the uniquely determined bounded harmonic function on $S$ with continuous boundary values $f(z)$ on $\partial S$ and by $h_{\varepsilon}$ the harmonic function on $S-\bar{V}_{\varepsilon}$ with continuous boundary values $f(z)$ on $\partial S$ and 0 on $\partial V_{\varepsilon}:|z|=\varepsilon$. Then the Stokes formula yields

$$
\begin{aligned}
\int_{\partial S} f(z) \frac{\partial e(z)}{\partial n} d s & =\int_{\partial\left(S-\bar{V}_{\varepsilon}\right)} h_{\varepsilon}(z) \frac{\partial e(z)}{\partial n} d s \\
& =\int_{S-\bar{V}_{\varepsilon}} \nabla h_{\varepsilon}(z) \cdot \nabla e(z) d x d y+\int_{S-\bar{V}_{\varepsilon}} h_{\varepsilon}(z) \Delta e(z) d x d y .
\end{aligned}
$$

Since the family of $h_{\varepsilon}$ is uniformly bounded on $S$, converges to $H_{f}^{S}$ uni- 
formly on each compact subset of $S \cup \partial S$ as $\varepsilon \rightarrow 0$,

$$
\int_{S}\left|\nabla\left(h_{s}-h_{\varepsilon^{\prime}}\right)(z)\right|^{2} d x d y=\int_{S}\left|\nabla h_{\varepsilon}(z)\right|^{2} d x d y-\int_{S}\left|\nabla h_{\varepsilon^{\prime}}(z)\right|^{2} d x d y
$$

for $\varepsilon>\varepsilon^{\prime}>0$ by setting $h_{\varepsilon}=0$ on $V_{s}$, and $e(z) P(z) d x d y$ is a finite measure on $\Omega$, the most right hand side of the above identity converges to

$$
\int_{S} \nabla H_{f}^{S}(z) \cdot \nabla e(z) d x d y+\int_{S} H_{f}^{S}(z) e(z) P(z) d x d y
$$

as $\varepsilon \rightarrow 0$ by the similar reasoning as in no. 1 . Therefore we have a generalization of (1):

$$
\int_{\partial S} f(z) \frac{\partial e(z)}{\partial n} d s=\int_{S} \nabla H_{f}^{S}(z) \cdot \nabla e(z) d x d y+\int_{S} H_{f}^{S}(z) e(z) P(z) d x d y
$$

3. We will give an upper estimate of the Dirichlet integral of the harmonic function $H_{1 / e}^{S}$ on $S$. Observe*) that $e \leqq H_{e}^{S}$. Since $H_{e}^{S}$ attains its minimum value on $\partial S$ we have

$$
\left(H_{e}^{S}(z)\right)^{-4} \leqq\left(\min _{\partial S} H_{e}^{S}\right)^{-4}=\left(\min _{\partial S} e\right)^{-4}=\max _{\partial S} e^{-4}
$$

on $S$. Applying the Dirichlet principle to functions $H_{1 / e}^{S}, 1 / H_{e}^{S}, H_{e}^{S}$, and $e$ on $S$, we have

$$
\begin{aligned}
\int_{S}\left|\nabla H_{1 / e}^{S}(z)\right|^{2} d x d y & \leqq \int_{S}\left|\nabla\left(1 / H_{e}^{S}(z)\right)\right|^{2} d x d y \\
& =\int_{S}\left(H_{e}^{S}(z)\right)^{-4}\left|\nabla H_{e}^{S}(z)\right|^{2} d x d y
\end{aligned}
$$

and similarly

$$
\int_{S}\left|\nabla H_{e}^{S}(z)\right|^{2} d x d y \leqq \int_{S}|\nabla e(z)|^{2} d x d y
$$

Therefore we have the following estimate:

$$
\int_{S}\left|\nabla H_{1 / e}^{S}(z)\right|^{2} d x d y \leqq\left(\max _{\partial S} e^{-4}\right) \int_{S}|\nabla e(z)|^{2} d x d y
$$

4. We next give an evaluation of the Dirichlet integral of $\log e$ on $\Omega-\bar{S}$. By the Stokes theorem we have

*) Here and also in no. 6 we use the fact that $e$ is subharmonic in $|z|<1$ by defining $e(0)=\lim \sup _{z \rightarrow 0} e(z)$. 


$$
\begin{gathered}
\int_{\partial \Omega} \frac{\partial e(z)}{\partial n} d s-\int_{\partial S}(1 / e(z)) \frac{\partial e(z)}{\partial n} d s=\int_{\partial(\Omega-\bar{s})}(1 / e(z)) \frac{\partial e(z)}{\partial n} d s \\
=\int_{\Omega-\bar{s}} \nabla(1 / e(z)) \cdot \nabla e(z) d x d y+\int_{\Omega-\bar{S}}(1 / e(z)) \Delta e(z) d x d y .
\end{gathered}
$$

If we set $S=\Omega$ in (1), then we have

$$
\int_{\partial \Omega} \frac{\partial e(z)}{\partial n} d s=\int_{\Omega} e(z) P(z) d x d y .
$$

In view of the identities $\nabla(1 / e(z)) \cdot \nabla e(z)=-|\nabla \log e(z)|^{2}$ and $(1 / e(z)) \Delta e(z)=$ $P(z)$, we deduce

$$
\begin{aligned}
\int_{\Omega-\bar{S}}|\nabla \log e(z)|^{2} d x d y= & \int_{\Omega-\bar{s}} P(z) d x d y-\int_{\Omega} e(z) P(z) d x d y \\
& +\int_{\partial S}(1 / e(z)) \frac{\partial e(z)}{\partial n} d s .
\end{aligned}
$$

5. The identity (4) shows that the Dirichlet integral of $\log e$ over $\Omega$ is essentially controlled by the integral of $(1 / e)(\partial e / \partial n)$ over $\partial S$. Therefore we have to study the behavior of the integral of $(1 / e)(\partial e / \partial n)$ over $\partial S$ as $\Omega-S$ exhausts $\Omega$, or, what amounts to the same, $\bar{S} \downarrow \varnothing$. For the purpose we consider two cases separately: $\lim _{\sup _{z \rightarrow 0}} e(z)=0$ and $>0$. First we consider the case $\lim \sup _{z \rightarrow 0} e(z)=0$, i.e. $\lim _{z \rightarrow 0} e(z)=0$. For every $t$ in $(0,1)$ consider the subregion $S_{t}: e(z)<t$ of $\Omega$, then $\bar{S}_{t} \downarrow \emptyset$ as $t \rightarrow 0$. Moreover from (1) it follows that

$$
\begin{aligned}
0 & \leqq \int_{S_{t}} e(z) P(z) d x d y=\int_{\partial S_{t}} \frac{\partial e(z)}{\partial n} d s \leqq \int_{\partial S_{t}}(1 / e(z)) \frac{\partial e(z)}{\partial n} d s \\
& =\frac{1}{t} \int_{\partial S_{t}} \frac{\partial e(z)}{\partial n} d s=\frac{1}{t} \int_{S_{t}} e(z) P(z) d x d y \leqq \int_{S_{t}} P(z) d x d y .
\end{aligned}
$$

Therefore the integral of $(1 / e)(\partial e / \partial n)$ over $\partial S_{t}$, which is nonnegative, converges to 0 as $t \rightarrow 0$ if $P(z) d x d y$ is a finite measure on $\Omega$.

6. Assume next that $\lim \sup _{z \rightarrow 0} e(z) \equiv a>0$. There exists a closed set $E$ thin at $z=0$ in $\Omega$ such that $e(z) \rightarrow a$ as $z \rightarrow 0$ with $z \notin E$ (cf. Brelot [4]). Then we may take a decreasing sequence $\left\{t_{m}\right\}$ in $(0,1)$ with $E \cap$ $\left\{z ;|z|=t_{m}\right\}=\emptyset$ for every $m$ and $\lim _{m \rightarrow \infty} t_{m}=0$. Applying (2) to the function $1 / e$ and the subregion $S_{m}: 0<|z|<t_{m}$ of $\Omega$ we have

$$
\begin{aligned}
\int_{\partial S_{m}}(1 / e(z)) \frac{\partial e(z)}{\partial n} d s= & \int_{S_{m}} \nabla H_{1 / e}^{S_{m}}(z) \cdot \nabla e(z) d x d y \\
& +\int_{S_{m}} H_{1 / e}^{S_{m}}(z) e(z) P(z) d x d y .
\end{aligned}
$$


The second term on the right hand side of the above equality is dominated by

$$
\left(\max _{\partial S_{m}} e^{-1}\right) \int_{S_{m}} e(z) P(z) d x d y,
$$

and moreover by (3) we have

$$
\begin{aligned}
\left(\int_{S_{m}} \nabla H_{1 / e}^{S_{m}}(z) \cdot \nabla e(z) d x d y\right)^{2} & \leqq \int_{S_{m}}\left|\nabla H_{1 / e}^{S_{m}}(z)\right|^{2} d x d y \cdot \int_{S_{m}}|\nabla e(z)|^{2} d x d y \\
& \leqq\left(\max _{\partial S_{m}} e^{-4}\right)\left(\int_{S_{m}}|\nabla e(z)|^{2} d x d y\right)^{2}
\end{aligned}
$$

Therefore we have

$$
\lim _{m \rightarrow \infty} \int_{\partial S_{m}}(1 / e(z)) \frac{\partial e(z)}{\partial n} d s=0 .
$$

7. Apply (4) to $S=S_{t}$ in the case of no. 5 or $S_{m}$ in the case of no. 6 and make $t \rightarrow 0$ or $m \rightarrow \infty$ accordingly. Then we obtain the following evaluation of the Dirichlet integral of $\log e$ on $\Omega$ :

TheOREM. For every density $P(z) d x d y$ on $\Omega$

$$
\int_{\Omega}|\nabla \log e(z)|^{2} d x d y=\int_{\Omega}(1-e(z)) P(z) d x d y .
$$

Here in the above equality it may happen $+\infty=+\infty$, which is exactly the case $P$ is not finite. As a direct consequence of this we obtain the following:

$$
\mathscr{D}_{1}=\mathscr{D}_{\subseteq D}
$$

\section{§2. Rotation free densities}

8. Consider a rotation free density $P(z) d x d y$ on $\Omega$, i.e. the density with $P(z)=P(|z|)$ on $\bar{\Omega}$. For every nonnegative integer $n$ we set $P_{n}(z)=P(z)$ $+n^{2} /|z|^{2}$, which is also a rotation free density on $\Omega$. Since the $P_{n}$-unit $e_{n}$, i.e. the unique bounded solution of $\Delta u=P_{n} u$ on $\Omega$ with the boundary values 1 on $\partial \Omega$, is also rotation free, it may be viewed as a function of $r$ in $(0,1]$. In other words, $e_{n}(r)$ may be considered as the unique bounded solution of

$$
\ell_{n} \psi(r) \equiv \ell_{n, P} \psi(r) \equiv \frac{d^{2}}{d r^{2}} \psi(r)+\frac{1}{r} \frac{d}{d r} \psi(r)-P_{n}(r) \psi(r)=0
$$

on $(0,1)$ with $e_{n}(1)=1$, where we follow the convention $P_{0}=P$ and $e_{0}=e$. 
We recall some of fundamental properties of $e_{n}$ (cf. [21], Imai [10]): For any $\rho \in(0,1]$,

$$
\frac{e_{n+1}(r)}{e_{n+1}(\rho)} \leqq \frac{e_{n}(r)}{e_{n}(\rho)} \quad(n=0,1, \cdots)
$$

for every $r$ in $(0, \rho]$; If we denote by $\psi^{\prime}$ the derivative $d \psi / d r$, then

$$
0 \leqq \frac{e_{n+1}^{\prime}(r)}{e_{n+1}(r)}-\frac{e_{n}^{\prime}(r)}{e_{n}(r)} \leqq \frac{1}{r} \quad(n=0,1, \cdots)
$$

on $(0,1]$; If $P \leqq Q$ on $\Omega_{\rho}: 0<|z|<\rho(0<\rho \leqq 1)$ for another rotation free density $Q(z) d x d y$ on $\Omega$, then

$$
\frac{e_{n}(\rho) e_{n+1}(r)}{e_{n+1}(\rho) e_{n}(r)} \leqq \frac{f_{n}(\rho) f_{n+1}(r)}{f_{n+1}(\rho) f_{n}(r)} \quad(n=0,1, \cdots)
$$

on $(0, \rho]$, where $Q_{n}(z)=Q(z)+n^{2} /|z|^{2}, f_{n}$ the $Q_{n}$-unit with the convention $f_{0}=f$ being $Q$-unit; The Picard principle is valid for $P$ if and only if

$$
\lim _{r \rightarrow 0} \frac{e_{1}(r)}{e_{0}(r)}=0 \text {. }
$$

In particular (7) was first shown by Imai [10; p. 182].

9. Consider a bounded solution $u$ of $L u=0$ on $\Omega$, i.e. $u \in \mathscr{B}_{P}$. In this and following nos. we will study the Dirichlet integral of $u / e$ in a neighborhood of $z=0$. For a continuous function $w$ on $\Omega$ the Fourier coefficients

$$
\left\{\begin{array}{l}
c_{0}(r)=c_{0}(r ; w)=\frac{1}{2 \pi} \int_{0}^{2 \pi} w\left(r e^{i \theta}\right) d \theta, \\
a_{n}(r)=a_{n}(r ; w)=\frac{1}{\pi} \int_{0}^{2 \pi} w\left(r e^{i \theta}\right) \cos n \theta d \theta \\
b_{n}(r)=b_{n}(r ; w)=\frac{1}{\pi} \int_{0}^{2 \pi} w\left(r e^{i \theta}\right) \sin n \theta d \theta
\end{array}\right.
$$

of $w$ are functions of $r$ alone in $(0,1)$. Since $u$ is a bounded solution of $L u=0$, the Fourier coefficients of $u$ satisfy that

$$
\begin{gathered}
\frac{d^{2}}{d r^{2}} c_{0}(r ; u)+\frac{1}{r} \frac{d}{d r} c_{0}(r ; u)=c_{0}\left(r ; \frac{\partial^{2} u}{\partial r^{2}}+\frac{1}{r} \frac{\partial u}{\partial r}\right) \\
=c_{0}\left(r ; \Delta u-\frac{1}{r^{2}} \frac{\partial^{2} u}{\partial \theta^{2}}\right)=P(r) c_{0}(r ; u)
\end{gathered}
$$




$$
\begin{aligned}
& \frac{d^{2}}{d r^{2}} a_{n}(r ; u)+\frac{1}{r} \frac{d}{d r} a_{n}(r ; u)=a_{n}\left(r ; \Delta u-\frac{1}{r^{2}} \frac{\partial^{2} u}{\partial \theta^{2}}\right) \\
& \quad=P(r) a_{n}(r ; u)-\frac{n}{r^{2}} b_{n}\left(r ; \frac{\partial u}{\partial \theta}\right)=\left(P(r)+\frac{n^{2}}{r^{2}}\right) a_{n}(r ; u),
\end{aligned}
$$

and similarly

$$
\frac{d^{2}}{d r^{2}} b_{n}(r ; u)+\frac{1}{r} \frac{d}{d r} b_{n}(r ; u)=\left(P(r)+\frac{n^{2}}{r^{2}}\right) b_{n}(r ; u) .
$$

Therefore they are bounded solutions of $\ell_{0} \psi=0$ or $\ell_{n} \psi=0$. For any fixed $\rho$ in $(0,1)$ we have

$$
\left\{\begin{array}{l}
c_{0}(r ; u)=\frac{c_{0}(\rho ; u)}{e(\rho)} e(r), \\
a_{n}(r ; u)=\frac{a_{n}(\rho ; u)}{e_{n}(\rho)} e_{n}(r), \\
b_{n}(r ; u)=\frac{b_{n}(\rho ; u)}{e_{n}(\rho)} e_{n}(r)
\end{array}\right.
$$

on $(0, \rho]$. Therefore the Fourier coefficients of $\partial u / \partial \theta$ may be represented in terms of $e_{n}$ in the following way:

$$
\begin{aligned}
& c_{0}\left(r ; \frac{\partial u}{\partial \theta}\right)=0, \\
& a_{n}\left(r ; \frac{\partial u}{\partial \theta}\right)=n b_{n}(r ; u)=n b_{n}(\rho ; u) \frac{e_{n}(r)}{e_{n}(\rho)},
\end{aligned}
$$

and similarly

$$
b_{n}\left(r ; \frac{\partial u}{\partial \theta}\right)=-n a_{n}(\rho ; u) \frac{e_{n}(r)}{e_{n}(\rho)}
$$

If we set $r=\rho$ then the Parseval identity yields that

$$
\sum_{n=1}^{\infty} n^{2}\left(a_{n}(\rho ; u)^{2}+b_{n}(\rho ; u)^{2}\right)=\frac{1}{\pi} \int_{0}^{2 \pi}\left(\frac{\partial}{\partial \theta} u\left(\rho e^{i \theta}\right)\right)^{2} d \theta<+\infty .
$$

Moreover from (5) it follows that

$$
\left\{\begin{array}{l}
a_{n}\left(r ; \frac{\partial u}{\partial \theta}\right)^{2} \leqq n^{2} b_{n}(\rho ; u)^{2} \frac{e_{1}(r)^{2}}{e_{1}(\rho)^{2}} \\
b_{n}\left(r ; \frac{\partial u}{\partial \theta}\right)^{2} \leqq n^{2} a_{n}(\rho ; u)^{2} \frac{e_{1}(r)^{2}}{e_{1}(\rho)^{2}}
\end{array}\right.
$$


for every positive integer $n$. Thus applying the Parseval identity to $\partial u / \partial \theta$ we have

$$
\begin{aligned}
& \int_{0}^{2 \pi} \int_{0}^{\rho}\left(\frac{1}{r} \frac{\partial}{\partial \theta} \frac{u\left(r e^{i \theta}\right)}{e(r)}\right)^{2} r d r d \theta \\
& \quad \leqq \frac{\pi}{e_{1}(\rho)^{2}} \sum_{n=1}^{\infty} n^{2}\left(a_{n}(\rho ; u)^{2}+b_{n}(\rho ; u)^{2}\right) \int_{0}^{\rho} \frac{1}{r}\left(\frac{e_{1}(r)}{e(r)}\right)^{2} d r
\end{aligned}
$$

for every $\rho$ in $(0,1)$.

10. The Fourier coefficients of $\partial(u / e) / \partial r$ are represented in terms of $e_{n}$ :

$$
\begin{aligned}
c_{0}\left(r ; \frac{\partial}{\partial r} \frac{u}{e}\right) & =c_{0}\left(r ; \frac{1}{e} \frac{\partial u}{\partial r}-\frac{e^{\prime}}{e^{2}} u\right)=\frac{1}{e(r)} \frac{d}{d r} c_{0}(r ; u)-\frac{e^{\prime}(r)}{e(r)^{2}} c_{0}(r ; u) \\
& =\frac{c_{0}(\rho ; u) e^{\prime}(r)}{e(r) e(\rho)}-\frac{e^{\prime}(r) c_{0}(\rho ; u) e(r)}{e(r)^{2} e(\rho)}=0, \\
a_{n}\left(r ; \frac{\partial}{\partial r} \frac{u}{e}\right) & =\frac{1}{e(r)} \frac{d}{d r} a_{n}(r ; u)-\frac{e^{\prime}(r)}{e(r)^{2}} a_{n}(r ; u) \\
& =\frac{a_{n}(\rho ; u) e_{n}(r)}{e(r) e_{n}(\rho)}\left(\frac{e_{n}^{\prime}(r)}{e_{n}(r)}-\frac{e^{\prime}(r)}{e(r)}\right),
\end{aligned}
$$

and similarly

$$
b_{n}\left(r ; \frac{\partial}{\partial r} \frac{u}{e}\right)=\frac{b_{n}(\rho ; u) e_{n}(r)}{e(r) e_{n}(\rho)}\left(\frac{e_{n}^{\prime}(r)}{e_{n}(r)}-\frac{e^{\prime}(r)}{e(r)}\right) .
$$

Then by (6) we have

$$
\begin{aligned}
a_{n}\left(r ; \frac{\partial}{\partial r} \frac{u}{e}\right)^{2} & \leqq\left(\frac{n a_{n}(\rho ; u) e_{n}(r)}{r e(r) e_{n}(\rho)}\right)^{2} \\
& \leqq \frac{n^{2} a_{n}(\rho ; u)^{2}}{r^{2} e_{1}(\rho)^{2}}\left(\frac{e_{1}(r)}{e(r)}\right)^{2}
\end{aligned}
$$

and similarly

$$
b_{n}\left(r ; \frac{\partial}{\partial r} \frac{u}{e}\right)^{2} \leqq \frac{n^{2} b_{n}(\rho ; u)^{2}}{r^{2} e_{1}(\rho)^{2}}\left(\frac{e_{1}(r)}{e(r)}\right)^{2}
$$

for every positive integer $n$, where $e_{0}=e$. Therefore applying the Parseval identity to $\partial(u / e) / \partial r$ we have

$$
\begin{aligned}
& \int_{0}^{2 \pi} \int_{0}^{\rho}\left(\frac{\partial}{\partial r} \frac{u\left(r e^{i \theta}\right)}{e(r)}\right)^{2} r d r d \theta \\
& \quad \leqq \frac{\pi}{e_{1}(\rho)^{2}} \sum_{n=1}^{\infty} n^{2}\left(a_{n}(\rho ; u)^{2}+b_{n}(\rho ; u)^{2}\right) \int_{0}^{\rho} \frac{1}{r}\left(\frac{e_{1}(r)}{e(r)}\right)^{2} d r
\end{aligned}
$$


for every $\rho$ in $(0,1)$. Thus in view of (9) and the above inequality the Dirichlet integral of $u / e$ on $\Omega_{\rho}$ satisfies the following:

$$
\begin{aligned}
\int_{\Omega_{\rho}}\left|\nabla \frac{u(z)}{e(z)}\right|^{2} d x d y & \\
& \leqq \frac{2 \pi}{e_{1}(\rho)^{2}} \sum_{n=1}^{\infty} n^{2}\left(a_{n}(\rho ; u)^{2}+b_{n}(\rho ; u)^{2}\right) \int_{0}^{\rho} \frac{1}{r}\left(\frac{e_{1}(r)}{e(r)}\right)^{2} d r
\end{aligned}
$$

for every $\rho$ in $(0,1)$.

11. Consider the function $v_{1}\left(r e^{i \theta}\right)=e_{1}(r) \cos \theta / e(r)$ on $\Omega$ and observe that $L\left(e_{1}(r) \cos \theta\right)=0$. Then $v_{1}$ is a bounded solution of $\hat{L} v=0$ on $\Omega$. Moreover from the fact that

$$
\left|\nabla v_{1}(z)\right|^{2} \geqq \frac{1}{r^{2}}\left(\frac{e_{1}(r)}{e(r)}\right)^{2} \sin ^{2} \theta
$$

it follows that

$$
\int_{\Omega_{\rho}}\left|\nabla v_{1}(z)\right|^{2} d x d y \geqq \pi \int_{0}^{\rho} \frac{1}{r}\left(\frac{e_{1}(r)}{e(r)}\right)^{2} d r
$$

for any $\rho$ in $(0,1)$, where $z=r e^{i \theta}$. Here note that

$$
\int_{\rho}^{1} r^{-1}\left(e_{1}(r) / e(r)\right)^{2} d r<+\infty
$$

for every $\rho$ in $(0,1)$.

12. In view of (11) the divergence of the integral of $r^{-1}\left(e_{1}(r) / e(r)\right)^{2}$ over $(0,1)$ implies the existence of a bounded solution of $\hat{L} v=0$ on $\Omega$ whose Dirichlet integral over a neighborhood of $z=0$ is infinite. Conversely assume that the integral of $r^{-1}\left(e_{1}(r) / e(r)\right)^{2}$ over $(0,1)$ is finite. Take an arbitrary bounded solution $v$ of $\hat{L} v=0$ on $\Omega$. Then the function $v e$ is a bounded solution of $L u=0$ on $\Omega$. In view of (10) the Dirichlet integral of $v=v e / e$ on a neighborhood of $z=0$ is finite. Therefore we obtain the following

THEOREM. Let $P(z) d x d y$ be a rotation free density on $\Omega$. Then the Dirichlet integral of every bounded solution of $\hat{L}_{P} v=0$ on a neighborhood of $z=0$ is finite if and only if

$$
\int_{0}^{1} \frac{1}{r}\left(\frac{e_{1}(r)}{e(r)}\right)^{2} d r<+\infty
$$


We have thus characterized $\mathscr{D}_{D} \cap$ \{rotation free densities\} completely: It is exactly the set of rotation free densities with (12). We feel characterizing the general $\mathscr{D}_{\circledast}$ is very difficult and we do not have even the foggiest idea at present.

\section{§3. Examples}

13. Consider the rotation free density $P(z) d x d y=|z|^{-2} d x d y$. The $P$ unit $d$ and the $\left(P(z)+1 /|z|^{2}\right)$-unit $d_{1}$ are given by $d(r)=r$ and $d_{1}(r)=r^{\sqrt{2}}$. Observe that

$$
\int_{\Omega}|\nabla \log d(z)|^{2} d x d y=2 \pi \int_{0}^{1} \frac{1}{r} d r=+\infty
$$

and yet

$$
\int_{0}^{1} \frac{1}{r}\left(\frac{d_{1}(r)}{d(r)}\right)^{2} d r=\int_{0}^{1} r^{2 \sqrt{2}-3} d r<+\infty
$$

Then from Theorem in no. 12 it follows that $P \in \mathscr{D}_{\mathscr{D}}-\mathscr{D}_{\Phi_{D}}$ and therefore

$$
\mathscr{D}_{\overparen{D}}<\mathscr{D}_{\mathbb{D}} \text {. }
$$

14. We will give a rotation free density belonging to $\mathscr{D}_{\mathfrak{R}}-\mathscr{D}_{\mathbb{D}}$. Let $0 \leqq \alpha<1 / 2$,

$$
\rho_{\alpha}=\max \left(\left(\frac{1}{\alpha}(5+2 \alpha)(1+2 \alpha)\right)^{1 / 2},\left(\frac{1}{8 \alpha}(3+2 \alpha)(1+2 \alpha)^{2}(1-2 \alpha)\right)^{1 / 4}\right)
$$

for $\alpha>0$, and $\rho_{0}=2$. Then the function

$$
F_{\alpha}(x)=1-\frac{1}{2}(5+2 \alpha)(1+2 \alpha) x^{-2}-\frac{1}{16}(3+2 \alpha)(1+2 \alpha)^{2}(1-2 \alpha) x^{-4}
$$

of $x$ in $\left[\rho_{\alpha},+\infty\right)$ satisfies that $F_{\alpha} \geqq 1-\alpha$ for $\alpha>0$ and $0 \leqq F_{0} \leqq 1$. Consider rotation free densities $P_{\alpha}(z) d x d y$ and $P_{\alpha 1}(z) d x d y$ defined by

$$
P_{\alpha}(z)= \begin{cases}\frac{1}{(1+2 \alpha)^{2}} F_{\alpha}(-\log |z|) \frac{(\log |z|)^{2}}{|z|} & \left(0<|z| \leqq \exp \left(-\rho_{\alpha}\right)\right) \\ P_{\alpha}\left(\exp \left(-\rho_{\alpha}\right)\right) & \left(\exp \left(-\rho_{\alpha}\right)<|z| \leqq 1\right)\end{cases}
$$

and $P_{\alpha 1}(z)=P_{\alpha}(z)+1 /|z|^{2}$. Observe that the function

$$
G_{\alpha}(r)=\frac{1}{1+2 \alpha} \frac{\log r^{-1}}{r}\left(1-\frac{1}{4}(3+2 \alpha)(1+2 \alpha)\left(\log \frac{1}{r}\right)^{-2}\right)
$$

of $r$ in $\left(0, \exp \left(-\rho_{\alpha}\right)\right)$ satisfies $G_{\alpha} \geqq 0$ and 


$$
\frac{d}{d r} G_{\alpha}(r)+G_{\alpha}(r)^{2}+\frac{1}{r} G_{\alpha}(r)=P_{\alpha}(r) .
$$

Then the function

$$
E_{\alpha}(r)=\exp \left(-\int_{r}^{\exp \left(-\rho_{\alpha}\right)} G_{\alpha}(t) d t\right)
$$

of $r$ in $\left(0, \exp \left(-\rho_{\alpha}\right)\right]$ is a bounded solution of

$$
\ell_{0, P_{\alpha}} \psi(r)=0 \text { with } E_{\alpha}\left(\exp \left(-\rho_{\alpha}\right)\right)=1 \text {. }
$$

Moreover it is easy to show the fact that $E_{\alpha}(r)\left(\rho_{\alpha} / \log r^{-1}\right)^{1 / 2+\alpha}$ is a bounded solution of $\ell_{1, P_{\alpha}} \psi(r)=0$ on $\left(0, \exp \left(-\rho_{\alpha}\right)\right)$ with the boundary values 1 at $r=\exp \left(-\rho_{\alpha}\right)$. Therefore the $P_{\alpha}$-unit $\left(P_{\alpha 1}\right.$-unit, resp.) $e_{\alpha 0}\left(e_{\alpha 1}\right.$, resp.) may be represented in terms of $E_{\alpha}$ on $\left(0, \exp \left(-\rho_{\alpha}\right)\right)$ as follows:

$$
\begin{aligned}
e_{\alpha 0}(r)= & E_{\alpha}(r) e_{\alpha 0}\left(\exp \left(-\rho_{\alpha}\right)\right) \\
& \left(e_{\alpha 1}(r)=E_{\alpha}(r)\left(\frac{\rho_{\alpha}}{\log r^{-1}}\right)^{1 / 2+\alpha} e_{\alpha 1}\left(\exp \left(-\rho_{\alpha}\right)\right), \text { resp. }\right) .
\end{aligned}
$$

By the above representation we have

$$
\frac{e_{\alpha 1}(r)}{e_{\alpha 0}(r)}=\frac{e_{\alpha 1}\left(\exp \left(-\rho_{\alpha}\right)\right)}{e_{\alpha 0}\left(\exp \left(-\rho_{\alpha}\right)\right)} \rho_{\alpha}^{1 / 2+\alpha}\left(\frac{1}{\log r^{-1}}\right)^{1 / 2+\alpha}
$$

and hence in view of (8) and Theorem in no. 12 we deduce $P_{\alpha} \in \mathscr{D}_{\mathscr{D}}(\alpha>0)$ and $P_{0} \in \mathscr{D}_{\mathfrak{R}}-\mathscr{D}_{\mathscr{D}}$, where $P_{0}=P_{\alpha}$ with $\alpha=0$.

15. Since the function $F_{\alpha}$ satisfies that $F_{\alpha} \geqq 1-\alpha$ for $\alpha>0$ and $F_{0}$ $\leqq 1$ on $\left[\rho_{\alpha},+\infty\right) P_{\alpha}$ satisfies that

$$
P_{\alpha}(z) \geqq \frac{1-\alpha}{(1+2 \alpha)^{2}} \frac{(\log |z|)^{2}}{|z|^{2}}
$$

for $\alpha>0$ and

$$
P_{0}(z) \leqq \frac{(\log |z|)^{2}}{|z|^{2}}
$$

on $0<|z| \leqq \exp \left(-\rho_{\alpha}\right)$, where $P_{0}(z)=P_{\alpha}(z)$ with $\alpha=0$. Observe that $\lim _{\alpha \rightarrow 0}(1-\alpha)(1+2 \alpha)^{-2}=1$. Then in view of (7) and Theorem in no. 12 the rotation free density $c|z|^{-2}(\log |z|)^{2} d x d y$ satisfies

$$
\begin{cases}c|z|^{-2}(\log |z|)^{2} \in \mathscr{D}_{\mathscr{D}} & \text { for } c \in[0,1), \\ c|z|^{-2}(\log |z|)^{2} \notin \mathscr{D}_{D} & \text { for } c \in[1,+\infty) .\end{cases}
$$


However $c|z|^{-2}(\log |z|)^{2} \in \mathscr{D}_{\Re}$ for every $c \in[0,+\infty)$. The relation (13) suggests the delicacy of the class $\mathscr{D}_{\mathscr{D}}$. It is not convex. It is known that $\mathscr{D}_{\Re}=\mathscr{D}_{\Re}$ is also not convex (cf. [23], Kawamura [15]). We have thus completed the classification as announced in the introduction:

$$
\mathscr{D}_{1}=\mathscr{D}_{\subseteq D}<\mathscr{D}_{\mathscr{D}}<\mathscr{D}_{\Re}=\mathscr{D}_{\mathfrak{P}}<\mathscr{D} \text {. }
$$

As for the last strict inclusion see e.g. [21].

\section{REFERENCES}

[1] A. Boukricha, Das Picard-Prinzip und verwandte Fragen bei Störung von harmonischen Räumen, Math. Ann., 239 (1979), 247-270.

[2] M. Brelot, Étude des l'équation de la chaleur $\Delta u=c(M) u(M), c(M) \geqq 0$, au voisinage d'un point singulier du coefficient, Ann. Sci. École Norm. Sup., 48 (1931), 153-246.

[ 3 ] - Sur le principe des singularités positives et la notion de source pour l'équation (1) $\Delta u(M)=c(M) u(M),(c \geqq 0)$, Ann. Univ. Lyon Sci. Math. Astro., 11 (1948), $9-19$.

[4] - On Topologies and Boundaries in Potential Theory, Lecture Notes in Math., Springer, 1971.

[5] C. Constantinescu und A. Cornea, Über einige Problem von M. Heins, Rev. Roumaine Math. Pures Appl., 4 (1959), 277-281.

[6] — Ideale Ränder Riemannscher Flächen, Springer, 1963.

[ 7 ] M. Godefroid, Sur un article de Kawamura et Nakai à propos du principe Picard, Bull. Sci. Math., 102 (1978), 295-303.

[8] K. Hayashi, Les solutions positives de l'équation $\Delta u=\mathrm{Pu}$ sur une surface de Riemann, Kōdai Math. Sem. Rep., 13 (1961), 20-24.

[ 9 ] M. Heins, Riemann surfaces of infinite genus, Ann. of Math., 55 (1952), 296-317.

[10] H. Imai, On singular indices of rotation free densities, Pacific J. Math., 80 (1979), $179-190$.

[11] H. Imai and T. Tada, Picard principle for rotation free densities on the Euclidean $N$-space $(N \geqq 3)$, Bull. Daido Inst. Tech., 13 (1978), 1-12.

[12] S. Itô, Martin boundary for linear elliptic differential operator of second order in a manifold, J. Math. Soc. Japan, 16 (1964), 307-334.

[13] M. Kawamura, Picard principle for finite densities on some end, Nagoya Math. J., 67 (1977), 35-40.

[14] - On a conjecture of Nakai on Picard principle, J. Math. Soc. Japan, 31 (1979), 359-372.

[15] — A remark on inhomogeneity of Picard principle, J. Math. Soc. Japan, 32 (1980), 517-519.

[16] M. Kawamura and M. Nakai, A test of Picard principle for rotation free densities II, J. Math. Soc. Japan, 28 (1976), 323-342.

[17] Z. Kuramochi, An example of a null-boundary Riemann surface, Osaka Math. J., 6 (1954), 83-91.

[18] A. Lahtinen, On the existence of singular solutions of $\Delta u=P u$ on Riemann surfaces, Ann. Acad. Sci. Fenn., 546 (1973).

[19] R. Martin, Minimal positive harmonic functions, Trans. Amer. Math. Soc., 49 (1941), 137-172. 
[20] M. Nakai, The space of nonnegative solutions of the equation $\Delta u=P u$ on a Rieman surface, Kōdai Math. Sem. Rep., 12 (1960), 151-178.

[21] — Martin boundary over isolated singularity of rotation free density, J. Math. Soc. Japan, 26 (1974), 483-507.

[22] —- A test for Picard principle, Nagoya Math. J., 56 (1974), 105-119.

[23] —- A remark on Picard principle, Proc. Japan Acad., 50 (1974), 806-808.

[24] — A test of Picard principle for rotation free densities, J. Math. Soc. Japan, 27 (1975), 412-431.

[25] — A remark on Picard principle II, Proc. Japan Acad., 51 (1975), 308-311.

[26] — Picard principle and Riemann theorem, Tôhoku Math. J., 28 (1976), 277292.

[27] —-, Picard principle for finite densities, Nagoya Math. J., 70 (1978), 7-14.

[28] — Strong Picard principle, J. Math. Soc. Japan, 32 (1980), 631-638.

[29] M. Ozawa, Some classes of positive solutions of $\Delta u=P u$ on Riemann surfaces, I; II, Kōdai Math. Sem. Rep., 6 (1954) ; 7 (1955), 121-126; 15-20.

[30] J. L. Schiff, Nonnegative solutions of $\Delta u=P u$ on open Riemann surfaces, J. Analyse Math., 27 (1974), 230-241.

[31] M. Šr, The Martin boundary for a linear elliptic second order operator, Izv. Akad. Nauk SSSR, 27 (1963), 45-60 (Russian).

[32] T. Tada, On a criterion of Picard principle for rotation free densities, J. Math. Soc. Japan, 32 (1980), 587-592.

\author{
Mitsuru Nakai \\ Department of Mathematics \\ Nagoya Institute of Technology \\ Gokiso, Showa, Nagoya 466 \\ Japan \\ Toshimasa Tada \\ Department of Mathematics \\ Daido Institute of Technology \\ Daido, Minami, Nagoya 457 \\ Japan
}

